\title{
E1 proceso de paz en Colombia y la política internacional
}

Los analistas de la vida política han venido reconociendo, desde hace algún tiempo, la internacionalización de los fenómenos politicos, económicos y sociales y, en algunos casos, su transnacionalizacion. Las teorías sobre la dependencia cedieron el paso a las que planteaban la interdependencia. Unas y otras se quedaron cortas en describir y analizar la compleja red de relaciones que se pretendía expresar con categorías tan comprehensivas ${ }^{1}$. Entre nosotros, este tipo de debate intelectual no ha formado parte significativa de la controversia política. Tan sólo referencias marginales y como para salvar la cara, son las que se pueden rastrear en las plataformas de los partidos o de sus facciones o en los Anales del Congreso o en las Memorias de los Ministros. Y de vez en cuando, una que otra confrontación. Sus repercusiones pronto desaparecen de eso que llaman ahora el discurso político, entiéndase, la retórica política.

Así las cosas, no es usual entre nosotros hacer esta suerte de conexiones, como la que voy a intentar hoy, entre nuestra política doméstica y la política internacional. Las gentes, aún las más iluminadas, prefieren examinarlas en forma desconectada y miran con una cierta sonrisa los ensayos para entrelazarlas. No es que por primera vez se dé entre nosotros ese vínculo entre política doméstica y política internacional. De suyo, siempre ha sido así. Lo que ocurre es que hoy es más patente, tiene implicaciones de honda envergadura y proyecta sus consecuencias, para bien o para mal, más allá del mandato electoral de la Administración Betancur.

Insisto. No es sólo la política doméstica de reconciliación nacional la que encuentra una dimensión internacional sino casi todos

\footnotetext{
'Al respecto, ver Fernando Cepeda Ulloa, La Teoría de la Dependencia: una ideologia paralizante, en Dependencia y Desarrollo, Anif, Bogotá, 1978, pp. 179197. Ver, también, algunas de sus columnas periodisticas: Partidos Transnacionales, EI Tiempo, 24 de junio de 1979; Nicaragua y la Opinión Pública Internacional, EI Tiempo, 22 de julio de 1979; La Oposición desde afuera, 8 de diciembre de 1979; El Terrorismo y la Transnacionalización de la Violencia, 2 de marzo de 1980.
} 
los aspectos de nuestro acontecer ${ }^{2}$. La economía colombiana no se entiende sin referencias al Banco Mundial, al Fondo Monetario, al Federal Reserve, al Banco Interamericano, para mencionar tan sólo una constelación de instituciones que tienen su sede en la capital norteamericana. De ello dan testimonio las frecuentes referencias a personalidades extranjeras en los discursos presidenciales: Clausen, Volcker, Knox, Ortiz Mena, Baker y, ahora, el profesor Hollis Chenery. Los viajes casi semanales del equipo económico a. Washington y Nueva York, son otro indicador de la creciente (y digámoslo, tal vez excesiva) internacionalización del proceso decisorio en materia económica ${ }^{3}$. Con la economía clandestina ocurre un fenómeno de internacionalización que no requiere explicación ${ }^{4}$. La Administración de Justicia, en casos graves, pasa también por los tribunales norteamericanos. $\mathrm{Y}$, en este caso, hay reciprocidad. En materia de ciencia y tecnología, en el contenido de los medios de comunicación masiva, en especial la televisión, en la obtención del capital y del know-how para la exploración y explotación de nuestros recursos mineros y energéticos, y así de los demás sectores. No estamos, pues, solos en el mundo. Vivimos en lo que se ha llamado una "aldea planetaria". Y el asunto clave no está en aislarse ni en conectarse sino en saber cómo participar más inteligentemente en el sistema internacional del cual somos miembros. En otras palabras, en cómó maximizar nuestras ventajas y minimizar nuestras desventajas. Vale decir, cómo insertarnos en el contexto internacional sin caer en la dependencia y sin sucumbir ciegamente a los encantos que ofrece el mundo desarrollado.

Debo recoger el hilo de esta exposición: La relación entre el proyecto de paz.interna y la política internacional. Voy a abocar el tema de una vez.

Preguntémonos: ¿Sería posible administrar con alguna probabilidad de exito un proyecto de paz interna, que busca incorporar a la lucha política desarmada a guerrillas de inspiración ideológica radical, con una política internacional hostil a la Unión Soviética, adversaria de la postura de Guba y Nicaragua en Gentroamérica e identificada con la línea del gobierno del Presidente Reagan tanto en el Istmo como en el resto del mundo? En mi opinión eso sería equi-

${ }^{2}$ Fernando Cepeda Ulloa, Prólogo en Gerhard Drekonja; Colombia: Politica Exterior, Bogotá, Universidad de los Andes-Fescol, 1982.

${ }^{3}$ Ver, por ejemplo, el informe presidencial después de su visita a Washington. (El Tiempo, 8 de abril de 1985, pág. primera), o los intercambios de correspondencia tanto con el Director del Fondo Monetario como con el Presidente del Banco Mundial. Para la referencia más reciente, ver El Espectador, 17 de junio de 1985, p. 2 (Sección Bogotá).

'Sobre las implicaciones internacionales y de seguridad del tráfico de drogas y su repercusión en Colombia, ver el discurso del Secretario de Estado, George Shultz; ante la Cámara de Comercio de Miami, 14 de septiembre de 1984: "Lazos entre el Terrorismo, las Drogas y algunos paises comunistas". 
valente a lograr la cuadratura del círculo. Tampoco bastaría una predisposición del gobierno colombiano para manejar estos factores del problema. Resulta indispensable que ellos mismos, oigase Cuba, Nicaragua, la Unión Soviética, exhiban una voluntad política similar y, claro está, que exista una capacidad política interna que con la habilidad y oportunidad necesarias sepa administrar la dimensión internacional del problema y su dimensión doméstica. Aclaro, de una vez por todas, y con todo el énfasis, que de ninguna manera subestimo los factores internos que han dado origen al conflicto guerrillero colombiano. Lejos de mí considerar que nuestra crisis - digo nuestra- se supera con una estrategia de política exterior. El conflicto es interno pero, sin duda, tiene una dimensión internacional que en ningún caso alcanza las proporciones de la crisis centroamericana, pero que no puede pasarse por alto si es que se quiere ver la realidad como verdaderamente es.

Una fugaz referencia a las políticas de Estados Unidos y de la Unión Soviética nos coloca en una perspectiva apropiada.

En términos generales, "Estados Unidos ha considerado siempre sus relaciones con el resto del Continente a la luz del enfoque de la seguridad", mientras que las naciones de América Latina prefieren hacer hincapié en las necesidades del desarrollo económico y en un trato más justo en materia comercial, financiera y de inversión ${ }^{5}$. Si se quiere, son dos caras de la misma moneda. La diferencia está en el énfasis. El tema da para muchas especulaciones. Una sobresaliente analista norteamericana hace un planteamiento más amplio. Margaret D. Hayes define así la posición de la gran nación del Norte: "Está en el interés nacional de Estados Unidos que exista en el hemisferio occidental, estados amigos, prósperos, con gobiernos estables y responsables que permitan la libre circulación de bienes y servicios en la región, que respeten la integridad política de sus vecinos; y, que no ofrezcan apoyo a los rivales de Estados Unidos en la escena política mundial".

No quiere decir esto que siempre este repertorio de intereses predominen o que no haya jerarquías entre ellos. La misma analista se encarga de hacer las correspondientes precisiones cuando explica el significado que tiene para Estados Unidos la adopción por parte de sus vecinos de políticas exteriores de alineamiento con sus adversarios en el nivel global. América Latina, en esa visión, quiéralo o no, gústele o no, es parte de la competencia Este-Oeste.

La crisis de cohetes de octubre de 1962 había dejado bien definido este aspecto con relación a Cuba y, obvio, a la Unión Soviética. Las reflexiones del Presidente Kennedy ante sus colabora-

${ }^{\top}$ Las relaciones América Latina-Estados Unidos en la segunda mitad de los años ochenta, en Cuadernos Semestrales, croe, No 15, Mréxico, 1984, p. 11. 
dores inmediatos cuando discutían lo que podría hacerse en República Dominicana ante la eventualidad de la desaparición de Trujillo, ilustra bien a las claras las verdaderas prioridades norteamericanas. Dijo el Presidente Kennedy: "Hay tres posibilidades en orden descendente de preferencia: un régimen democrático decente, la continuación del régimen de Trujillo, o un régimen castrista. Debemos buscar lo primero, pero realmente no podemos renunciar al segundo hasta tanto no estemos seguros de que podemos evitar el tercero"b.

La Unión Soviética, que es finalmente, la razón de ser de la estrategia de seguridad norteamericana en el hemisferio (así como en otros continentes), reconoce que esta región del mundo forma parte de la "esfera de influencia" norteamericana y se permite apenas incursiones que no desborden el límite de tolerancia de Washington ${ }^{7}$. Con todo, el surgimiento de Cuba como un país que, con la ayuda soviética, rompió la hegemonía norteamericana en el Continente, ha colocado las cosas en una nueva luz ${ }^{8}$. Cuba y la Unión Soviética no han coincidido en su evaluación de las potencialidades revolucionarias de América Latina ni en las estrategias para tramitarlas. Los sovietólogos que analizan la política soviética para Latinoamérica coinciden en este diagnóstico y establecen varias etapas en la evolución de esa difícil relación: desacuerdo con autonomía cubana para fomentar movimientos guerrilleros, sería una primera fase; desacuerdo con predominio de la tesis soviética de no fomentar activamente esos movimientos; y reactivación de la tesis cubana a raíz del triunfo del sandinismo en Nicaragua en julio de $1979 \mathrm{y}$ de las oportunidades que ofrecería la crisis centroamericana. Según algunos, esta coyuntura habría dado la razón. a la tesis cubana $y$, en consecuencia, habría inducido un cambio en la actitud soviética para tornarla activamente favorable a los movimientos

Margaret D. Hayes, Understanding United States Policy Toward Latin America. Mimeo, ponencia presentada al Seminario "Zona de Paz: hacia una Alternativa de Seguridad Regional para América Latina", Santiago de Chile, 5, 6 y 7 de junio de 1985, pp. 2 y 8-9.

"Las apreciaciones sobre la politica exterior soviética están basadas en algunos de los trabajos de los sovietólogos Robert S. Leiken, Tiri Valenta, Edmé Domínguez, Isabel Torrent, Augusto Varas, Carlos Pérez Llana. El autor ha hecho referencias a este asunto, así: Objetivos soviéticos en el Caribe, El Tiempo, Bogotá, 16 de febrero de 1982, p. 4.

"El análisis más completo al respecto es el de Robert S. Leiken, "Soviet Strategy in Latin America". Ver tambiên Susan K. Purcell, The cuban factor in the Central American Conflict. Mimeo, presentado a la conferencia sobre "La crisis centroamericana y sus implicaciones internacionales, organizada por el Instituto Internacional de Estudios Estratégicos de Londres y El Colegio de México, Toluca, México, 20 de mayo de 1985. Al respecto, también conviene consultar el trabajo del General (R) Alvaro Valencia Tovar "Estrategia Soviética en el Caribe", Occasional Papers, Institute of Interamerican Studies, University of Miami, febrero de 1984. 
guerrilleros latinoamericanos. Según otros, este cambio solamente habría ocurrido en la opinión de expertos y de funcionarios pero jamás en la cúpula decisoria soviética, la cual mantendría una actitud que se denomina de "pragmatismo flexible" frente a Latinoamérica ${ }^{9}$.

Si se le da crédito a esta última postura -y yo me matriculo en esa interpretación-, la Unión Soviética estaría interesada en jugar un papel menor en esta parte del mundo, reconociendo la esfera de influencia norteamericana. Lo política latinoamericana estaría condicionada a un interés más alto para la Unión Soviética, que sería su propia relación con los Estados Unidos, y matizada por su relación bilateral con cada uno de los países y las especificas coyunturas políticas. Esta evaluación hace mucho más complejo el entendimiento de los objetivos y de las estrategias soviéticas en cada coyuntura pero aconseja, eso sí, rechazar por simplistas e inapropiadas afirmaciones que alegremente -o histéricamente- proclamen la llegada de los soviéticos para construir el socialismo sin libertad ${ }^{10}$. Pero de otro lado, hay que comprender que la presencia de Cuba Comunista ha introducido, en forma ineludible, la perspectiva Este-Oeste cuantas veces se plantea un cambio político por la vía revolucionaria ${ }^{11}$.

Debe entenderse que la Unión Soviética tiene dos líneas de política exterior, la de las relaciones Estado-Estados y la de las relaciones Partido-Partidos ${ }^{12}$. No siempre coinciden, pero siempre prevalecen las primeras, las Estado-Estados. Sobra decir que los Partidos Comunistas locales presionan, con frecuencia, la revalorización de la vía armada. Son más ideológicos que pragmáticos. Son más ortodoxos. Más papistas que el Papa.

Conviene preguntarse: ¿la posición de la Administración Reagan al exacerbar la dimensión Este-Oeste de los conflictos internacionaIes ha inducido un nuevo comportamiento de la Unión Soviética? Los que siguen con cuidado estas materias dicen que ello no ha sido

'Isabel Turrent, La Unión Soviética y América Latina. Mimeo, ponencia presentada a la Conferencia sobre "La crisis centroamericana y sus implicaciones internacionales", p. I4.

${ }^{10}$ Algunas de las presentaciones hechas en el Seminario sobre la Penetración Soviético-Cubana en Centroamérica y en el Caribe (Costa Rica, febrero de 1984), apuntaban en esa dirección, así como las interpretaciones de algunos de Ios miembros del equipo del Presidente Reagan en la campaña y en la primera parte de su gobierno. Ver Juan Manuel del Aguila, Caribbean Basin Vulnerabilities and Soviet Guban Penetration, febrero de 1984; Ernst Halperin, The Cuban Role in Gentral America and the Caribbean, febrero de 1984. Halperin afirma, por ejemplo, con respecto a las ambiciones de Moscú: "Desde 1959 existe amplia evidencia que muestra que sus ambiciones no estaban limitadas a Guba. Su objetivo final: expulsar a los Estados Unidos de toda América Latina", p. I.

"Susan K. Purcell, op. cit., p. I9.

2Isabel Turrent, op. cit., p. 4 y ss. 
así. Uno de ellos afirma: "Las relaciones URss-América Latina han mantenido sus características centrales durante la primera administración del Presidente Reagan en los Estados Unidos, éstas son, un bajo perfil confrontacional en el plano militar, fuerte apoyo político a países en peligro de agresión, y uso de la ayuda económica como instrumento de influencia en las políticas exteriores de los países latinoamericanos"13.

El caso de la Isla de Granada ilustra bien la naturaleza de esta compleja relación entre Estados Unidos y la Unión Soviética. Muestra hasta dónde puede llegar la Unión Soviética y cuál es el límite de tolerancia de los Estados Unidos. Y, más importante todavía, hasta qué punto Cuba y la Unión Soviética respetan la concepción de seguridad norteamericana. La Unión Soviética penetra hasta donde las circunstancias lo permitan, pero retrocede o se ausenta si es necesario frente a una acción decidida norteamericana. Las crisis de los cohetes en 1962 y Granada así lo indican. La prudencia frente a Nicaragua, también. El precario compromiso con Allende, aunque en otro contexto, es otro ejemplo. Estamos, pues, ante una política más sofisticada que la sugerida por la retórica.

A mi entender, el Presidente Betancur hizo después de la campaña presidencial una lectura de la coyuntura internacional y de sus interrelaciones con la situación colombiana muy semejante a la que, se me antoja, había hecho el ex Presidente López Michelsen antes de iniciar su campaña cuando propuso tanto una política de Paz como la afiliación del partido liberal a la Internacional Socialista, o sea, una política de paz con soporte en una nueva política exterior. Una similar a la que en ese momento propiciaba la Internacional Socialista en Centroamérica, diferente, por supuesto, de la del Departamento de Estado ${ }^{14}$. Esa lectura del contexto internacional colocaba a Colombia ante el dilema de enmarcarse en la guerra fría, en el conflicto Este-Oeste, con el riesgo de escalar a niveles impensables el conflicto interno colombiano o salirse de ese esquema diabólico para solucionar en sus términos, pero sin desconocer la dimensión internacional el conflicto guerrillero, Betancur como Presidente Electo se apropia este diagnóstico y, luego, le aplica su propia estrategia.

En varias ocasiones y en diferentes escritos he recogido el itinerario de lo que he denominado la Estrategia de Paz, la cual tiene, debo reiterarlo, una dimensión doméstica, una dimensión interna-

${ }^{23}$ Augusto Varas, La Unión Soviética frente a América Latina. Mimeo, ponencia presentada al Seminario Internacional "Zona de Paz: hacia una alternativa de Seguridad Regional para América Latina", Santiago de Chile, 5, 6 y 7 de junio, 1985, p. 7.

${ }^{14}$ Sobre el papel de la Internacional Socialista, consultar Edgar Jiménez Cabrera, La estrategia socialdemócrata en América Latina, en Cuadernos Semestrales, ut supra, nota 5, pp. 235-252. 
cional, otra retórico-sicológica y otra económica y social. Aquí me estoy ocupando y, sólo superficialmente, del aspecto internácional ${ }^{15}$.

El cambio de actitud frente al proyecto de construcción de la represa de Urrá con ayuda de la Unión Soviética constituye, sin duda, la gran señal en virtud de la cual el Presidente Electo anuncia un trato diferente con Ia Unión Soviética al insinuado durante su campaña. Luego vendrá el cambio de posición frente al conflicto de Malvinas, la afiliación al Movimiento de los No-Alineados (la cual, entre otras funciones, cumplió la de reestablecer de hecho las relaciones diplomáticas con Fidel Castro, entonces presidente de ese Movimiento), la declaración en el sentido de que Colombia no es un país satélite de los Estados Unidos, el distanciamiento con respecto a la política exterior norteamericana, el regaño al Presidente Reagan con ocasión de su visita a Bogotá y, en enero de 1982, la creación del grupo de Contadora, es decir, la protocolización de una estrategia de búsqueda de una paz negociada en América Central. En esencia, un esquema de diplomacia regional dirigido a encontrar una concepción de seguridad diferente a la norteamericana en una parte del hemisferio especialmente simbólica para los intereses de Estados Unidos, en la cual se había producido, en menos de treinta años, dos triunfos de movimientos guerrilleros: el castrismo y el sandinismo, ambos visualizados como desafíos a la hegemonía norteamericana en el área.

El Grupo de Contadora es la herramienta en virtud de la cual la Administración Betancur tramita la esencia de su nueva concepción de política exterior, coherente con el esquema de reconciliación doméstica, el cual se puede sintetizar en las siguientes categorías: rechazo o abandono de la actitud anticomunista; respeto al pluralismo ideológico como una expresión del principio de autodeterminación de los pueblos; condena al intervencionismo de potencias o países en conflictos internos; respeto a los procesos de reconciliación interna; negación del conflicto Este-Oeste, como explicativo de la situación centroamericana.

De un solo golpe, se bloqueaba la centroamericanización del conflicto colombiano. Los indicios comprobados de intervención cubana en el movimiento guerrillero colombiano que dio lugar a la suspensión de las relaciones diplomáticas (23 de marzo de 1981) y la

${ }^{28}$ Una presentación extensa aparece en Fernando Cepeda Ulloa, Contadora: el Proceso de Paz en Colombia y Centroamérica, en Revista Nacional de Agricultura, No 870, marzo de 1985, pp. 80-102.

Gabriel Silva Luján, Política Exterior, Continuidad o Ruptura, Reseña de un Debate, Bogotá, Centro de Estudios Internacionales (Universidad de los Andes) y CEREC, 1985, recoge las diversas opiniones que un grupo de expertos, colombianos y extranjeros, emitieron sobre la política internacional de la Administración Betancur, Para : el tema de este trabajo es especialmente relevante el capitulo Iv: La. Politica Exterior y el Proceso Político Nacional, pp. 95-116. 
agudización de los conflictos con Nicaragua y sus previsibles consecuencias, quedan relegados y amortiguados. El ambiente para las etapas subsiguientes a la Amnistía se torna mucho más positivo. La credibilidad del Presidente y de su Estrategia de Paz tienen ahora un aval en el proceso de Contadora. Pero también un nuevo factor que va a influir muchos aspectos del proceso de paz. La seriedad del compromiso colombiano en Centroamérica vía Contadora garantiza el compromiso de paz interno. Y viceversa. En adelante no se ve cómo podría sostenerse una política de paz contradictoria. Una para 'Centroamérica y otra para Colombia. El diálogo y la negociación que se ofrecen en Centroamérica pronto se hacen imperativos en Colombia. Quienes creían que la Amnistía era el instrumento fundamental y casi definitivo del proceso de paz se engañaron. La Amnistía no es sino un gesto. Y ni siquiera el más importante, aunque sí indispensable. Si hablamos logrado bloquear la centroamericanización del conflicto, ahora adoptamos la solución centroamericana, la que estábamos propiciando en Centroamérica, es decir, la negociación con los grupos guerrilleros. Así, el proyecto de Contadora adquiría más credibilidad. Había sido impensable estimular la negociación en Centroamérica y negarla en Colombia.

Al hacer estas reflexiones no sobra reparar en el hecho de que Colombia es el único país del Grupo Contadora que está administrando un proceso de pacificación similar al que se reclama para los países centroamericanos. En consecuencia, lo que se haga en Contadora y lo que se haga en Colombia tiene que exhibir alguna coherencia, porque de otra manera se perderia toda credibilidad. Contadora tiene, pues, para Colombia un significado muy superior al que puede tener, desde el punto de vista de la política interna, para Venezuela o México o Panamá. Apoyar Contadora es apoyar el proceso de paz en Colombia. Los dos esquemas, por decir lo menos, van de la mano.

Es bien probable que el ritmo de Contadora marque el ritmo deI proceso de paz en Colombia. No se ve cómo podríamos propiciar la estrategia kissingeriana de los dos carriles, o sea del plomo y las negociaciones internamente sin respaldarla en Centroamérica. Tampoco se entiende cómo podríamos recomendar allá unos mecanismos de verificación del cese de fuego y del desarme sin adoptarlos aquíl ${ }^{16}$.

La estrategia doméstica de paz ha sido planteada como un modelo tanto por el precandidato demócrata Hart como por los guerrilleros centroamericanos. No es sorprendente que el Frente Farabundo Martí hubiera invitado al Presidente Betancur para servir

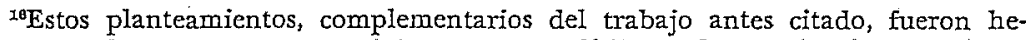
chos por el autor tanto en México como en Chile en los Seminarios mencionados en las notas anteriores.
} 
como mediador en sus negociaciones con el Presidente Duarte en octubre de 1984. Y es apenas natural que el Secretario de Estado Shultz y el Presidente Duarte descalifiquen la validez para Centroamérica del experimento colombiano ${ }^{17}$.

Las negociaciones que llevaron a suscribir Acuerdos de Paz con los grupos guerrilleros así en marzo de 1984 como en agosto del mismo año han sido presentadas como un paradigma, como un modelo de solución para Centroamérica. El empantanamiento del proceso en Golombia contribuye a dificultarlo en Centroamérica. Y viceversa.

Si se quiere saber por qué hay un éxito mayor y reconocido en las negociaciones con las Fuerzas Armadas Revolucionarias de Colombia (FARC) hay que reparar en sus vínculos con Cuba y la Unión Soviética y con el partido comunista colombiano. Si se examina este fenómeno a la luz de lo que arriba se dijo sobre la politica exterior de Ia Unión Soviética, todo es más claro. No ocurre exactamente lo mismo con otros grupos guerrilleros sin que se nieguen sus vinculos internacionales, pero que en su caso son de naturaleza bien diferente. Por lo menos así parece.

FARC y $M-19$ son dos versiones bien distintas, desde el punto de vista internacional, del movimiento guerrillero. Las FARG pertenecerían a lo que los especialistas en estos temas llaman la guerrilla de primera generación. El M-19 a lo que clasifican como guerrilla de segunda generación. Examinemos el perfil propio de esta última, siguiendo el análisis de César Sereseres, profesor de la Universidad de California y asesor del Departamento de Estado: "El nuevo modelo de la guerrilla ofrecería las siguientes caracteristicas:

a) Las organizaciones guerrilleras han desarrollado bases de apoyo más amplias tanto en el nivel doméstico como en el internacional.

b) Las tácticas de lucha han ido más allá de la teorfa del foco revolucionario de los años sesenta y ahora responde a una estrategia modificada de guerra prolongada.

c) Las organizaciones guerrilleras han desarrollado una "política exterior" elaborada y sofisticada que se extiende a otras naciones latinoamericanas, a Europa y a los Estados Unidos.

d) Los gobiernos, los partidos políticos, las iglesias, los sindicatos, los grupos de presión y otros actores externos a la región proporcionan recursos y con frecuencia están involucrados en forma directa.

${ }^{17}$ Las referencias bibliográficas pertinentes pueden ser consultadas en el estudio citado en la nota 15 . Para las opiniones del Secretario de Estado, George Shultz, ver: "Paz a la colombiana no es aplicable a Centroamérica", Bogotá, E1 Tiempo, 24 de febrero de 1985, pp. 1 y I5. 
Estas nuevas características de la guerra revolucionaria en Centroamérica -comenta Sereseres-dan a entender que la así llamada "guerra interna" ha sido internacionalizada hasta tal punto que el conflicto puede ser determinado desde más allá de las fronteras del pais en conflicto"18.

Cabe preguntarse hasta qué punto la nueva estrategia de contrainsurgencia planteada públicamente por el gobierno norteamericano, sometida a debate y aprobación del Congreso y a la controversia en la opinión pública, responde a una búsqueda de simetría frente a este nuevo estilo de guerrilla. Sin duda, constituye una innovación de mucha significación la de anunciar y financiar a la luz del sol grupos de contrainsurgencia en Nicaragua o en Afganistán. Se legitima así la internacionalización de los conflictos "internos" $y$, definitivamente, se les enmarca en el conflicto Este-Oeste, con todas sus consecuencias. Me atrevería a decir que la era de los conflictos internos, lo que Eckstein analizó como "internal war", está desapareciendo del escenario global. Ahora tendremos guerrillas de lado y lado, con sus jefes de relaciones públicas, sus doctrinas de justificación, sus héroes, su repertorio de soportes a lo largo y ancho del mundo. Son cambios que debemos tomar en consideración si nos proponemos entender lo que ocurre alrededor de nosotros.

Para abundar en la postura soviética conviene citar in extenso las conclusiones a que llega el sovietólogo chileno Profesor Augusto Varas al analizar el tema "La Unión Soviética frente a América Latina", en el reciente seminario auspiciado por CEPAL-FLACSO y RIAL en Santiago de Chile, durante la segunda semana de junio del presente año. Luego de hacer referencia a los casos de los partidos comunistas de Uruguay, Honduras, Venezuela y República Dominicana, dice el Profesor Varas: "Sin embargo, los desarrollos más significativos al respecto han sido las evoluciones al interior del Partido Gomunista colombiano. Este ha modificado de manera importante su política frente a los cambios a introducir en la sociedad colombiana y en su apreciación de la administración de Betancur. En primer lugar, el PCC ha reconocido que la política exterior de Betancur es un progreso hacia una mayor independencia de Ios Estados Unidos. En segundo Iugar, ha aceptado el cese del fuego y la ley de amnistía promulgada por el gobierno. Sin embargo, este acuerdo de apoyar el cese del fuego entre las Farc (Fuerzas Armadas Revolucionarias de Colombia) y el gobierno, terminó con la renuncia al partido del único senador suplente que tenía el $P G$, senador

${ }^{28}$ Gésar D. Sereseres, Changing Guerrilla Warfare Strategies in Central America. Mimeo, ponencia presentada a la conferencia sobre "La crisis centroamericana y sus implicaciones internacionales", organizada por el Instituto Internacional de Estudios Estratégicos de Londres y el Colegio de México, Toluca, México, 20 de mayo de 1985. 
Humberto Criales, en el pleno realizado antes de Semana Santa de 1984. El senador expresó sus profundas reservas sobre la efectividad del acuerdo en la medida que, según su opinión, las condiciones económicas y sociales del país impiden al presidente Betancur realizar sus promesas de reformas estructurales. A pesar de este fuerte golpe a las posiciones del PC en el medio político colombiano, éste aceptó el costo, toda vez que la triple relación gobierno colombiano, gobierno soviético y PCC, ha comenzado a orientarse en otra dirección.

La actitud de la URSS al respecto ha sido categórica, en apoyo de esta nueva orientación. Así, la delegación soviética al xrv Congreso del PC colombiano -celebrada en noviembre de 1984, con posterioridad a la aceptación de la política de pacificación de Betancur y de la consecuente renuncia de su único senador-, encabezada por R. I. Kosolapov, miembro del Comité Central del pcus y director de la revista teórica del Comité Central del PGUS, "KOMUnIST", entregó un mensaje a los congresales indicándoles el apoyo del pcus a Ia lucha del PGC, "por el desarrollo independiente de Colombia en el camino de la democracia y el progreso"19.

La verdad es que el proceso de paz no va a quedar consolidado durante la presente Administración. La empresa hay que continuarla. Y para ello se requieren todas las luces de la inteligencia y de la información sobre lo que acontece en el mundo y la manera como nos afecta. Estas reflexiones constituyen un modestísimo aporte en esa tarea. Hay quienes creen que la política exterior puede sufrir cambios sustanciales y no la refieren al proceso de paz. Postulo que eso es un error. Otros piensan que la estrategia interna de paz puede sufrir modificaciones significativas sin que se afecte la política exterior. Creo que es otro error. Un entendimiento cabal de la dimensión internacional del proceso de paz es útil para la formulación de las estrategias liberales del inmediato futuro y los respectivos ajustes.

${ }^{19}$ Augusto Varas, op. cit., p. 16. 